\title{
An Inter-Arrival Hyper-Exponential Machine Repairman problem with Two Heterogeneous Repairmen
}

\author{
Mahdy . S. EL-Paoumy \\ Statistics Department, Faculty of Commerce, AL- Azhar University, Girls` Branch, Egypt \\ E- mail: drmahdy_elpaoumy@yahoo.com
}

\begin{abstract}
This paper aims to treat the analytical solution of the truncated inter-arrival hyper-exponential machine interference queue $: H_{r} / M / 2 / k / N$ in case of $r$ branches with two heterogeneous repairmen. Our research treats the general case for the value of $r, k$ and $N$ considering the discipline FIFO with a more general condition. In the end we study some special cases.
\end{abstract}

Keywords: Heterogeneous repairmen; machine interference; inter-arrival hyperexponential

\section{1- Introduction :}

The truncated inter-arrival hyper-exponential machine interference queue: $H_{r} / M / c / k / N$ with balking and reneging treated by Shawky [ 9 ], George et al. [ 3 ] considered multiserver system of the type $G I / M / r$ where the coefficient of variation of inter-arrival times is greater than 1, and derived two simple approximations for the steady state average queueing time. Gupta [ 5 ] treated numerically the inter-arrival hyper-exponential queue: $H_{r} / M / 1 / m$ with state dependent arrival and service rates, and Abou-AlAta [1] treated the analytical solution of the truncated inter-arrival hyper-exponential machine interference queue : $\mathrm{H}_{2} / \mathrm{M} / \mathrm{c} / \mathrm{m} / \mathrm{m}$ with both balking and reneging in case homogeneous servers. The present paper treats the analytical solution of the truncated inter- arrival hyper-exponential machine interference queue: $H_{r} / M / 2 / k / N$ with two heterogeneous repairmen using a recurrence relation. Also some special cases are obtained.

\section{2 - Model Description:}

As in Goyal [4 ], the arrival channel consists of $r$ independent branches. A machine arriving for service joins the $i^{t h}$ branch with a fraction $\sigma_{i}$ of the time on the average, so that $\sum_{i=1}^{r} \sigma_{i}=1$. Only one machine can occupy any one of the branches at a time and if a machine is present in any one of the branches, the arrival channel is busy and no other machine can enter any other branch. The machine in the $i^{\text {th }}$ branch joins the system ( queue or service) with rate $\lambda_{i}$ per unit time. Finite source ( population) of $N$ machines, finite space with capacity $k$ machines $(k<N)$, and two heterogeneous servers( repairmen) are assumed. The service times are identical exponential random variables with $\operatorname{rates} \mu_{1}$, and $\mu_{2}\left(\mu_{1}>\mu_{2}\right)$.

An arriving machine may find :

(i) All repairmen engage, then it waits in a line in order of arrival, the machine at the top of the queue occupied the repairman that falls vacant first.

(ii) Only one of the repairmen free, so it occupies the free repairman. 
(iii) All repairmen free, thus it chooses repairman I with probability $\pi_{1}$, repairman II with probability $\pi_{2}$, such that, $\pi_{1}+\pi_{2}=1$. The classical queue discipline assumes that $\pi_{1}=\pi_{2}=\frac{1}{2}$. Saaty [ 12 ] took $\pi_{1}$ to be $\mu_{1}\left(\mu_{1}+\mu_{2}\right)^{-1}$ and $\pi_{2}$ to be $\mu_{2}\left(\mu_{1}+\mu_{2}\right)^{-1}$.

Gumble [ 6 ] has studied the many server ( heterogeneous) queue but under the classical queue discipline and Krishnamoorthi [ 8 ] has studied Poisson- queue with two heterogeneous servers with different queue disciplines.

\section{3-The Steady- State equations and their solution}

Define the equilibrium probabilities:

$p_{0,0, s}=$ prob. there is no machine in the system and $s^{t h}$ arrival branch occupied the next arriving machine\},

$p_{1,0, s}=$ prob. $\left\{\right.$ there is one machine in repairman Iand $s^{t h}$ arrival branch occupied the next arriving machine\},

$p_{0,1, s}=$ prob. $\left\{\right.$ there is one machine in repairman IIand $s^{\text {th }}$ arrival branch occupied the next arriving machine\},

$p_{n, s}=$ prob. $\left\{\right.$ there are $n$ machines in the system and $s^{\text {th }}$ arrival branch occupied the next arriving machine $\}, n=2 ., 3, . . k ; \mathrm{s}=1,2, \ldots r$.

Also, $p_{0, s}=p_{0,0, s} \quad, \quad p_{1, s}=p_{1,0, s}+p_{0,1, s}$ and $p_{2, s}=p_{1,1, s}$.

Consequently, the steady-state probability difference equations are:

$$
\begin{aligned}
& \quad N \lambda_{i} p_{0,0, i}=\mu_{1} p_{1,0, i}+\mu_{2} p_{0,1, i} \\
& \left\{(N-1) \lambda_{i}+\mu_{1}\right\} p_{1,0, i}=\sigma_{i} \pi_{1} \sum_{s=1}^{r} N \lambda_{s} p_{0, s}+\mu_{2} p_{1,1, i}, i=1(1) r \\
& \left\{(N-1) \lambda_{i}+\mu_{2}\right\} p_{0,1, i}=\sigma_{i} \pi_{2} \sum_{s=1}^{r} N \lambda_{s} p_{0, s}+\mu_{1} p_{1,1, i}, i=2(1) r \\
& \left\{(N-n) \lambda_{i}+\mu\right\} p_{n, i}=(N-n+1) \sigma_{\mathrm{i}} \sum_{s=1}^{r} \lambda_{s} p_{n-1, s}+\mu p_{n+1, i}, n=2,3, \ldots, k-1 \\
& \left\{(N-k) \lambda_{i}+\mu\right\} p_{k, \mathrm{i}}=(N-k+1) \sigma_{i} \sum_{s=1}^{r} \lambda_{s} p_{k-1, s}+(N-k) \sigma_{i} \sum_{s=1}^{r} \lambda_{s} p_{k, s} \\
& \text { where : } \quad \mu=\mu_{1}+\mu_{2} .
\end{aligned}
$$

Summing up equations (1) - (5)over $\mathrm{i}$, and adding the results obtained for $n=1,2, \ldots k-1$, we get

$$
\sum_{s=1}^{r} p_{n+1, s}=(N-n+1) \sum_{s=1}^{r} \rho_{s} p_{n, s}, n=1,2, \ldots, k-1
$$

where $\rho_{s}=\frac{\lambda_{s}}{\mu}$.

From (5) and (6) for $n=k-1$, we have

$$
\left[(N-k) \rho_{i}+1\right] p_{k, i}=\sigma_{i} \sum_{s=1}^{r}\left[(N-k) \rho_{s}+1\right] p_{k, s},
$$


It is easy to verify that the determinate formed by the coefficients of $p_{k, i}, i=1,2, \ldots r$ is zero and therefore we can solve equation(7) for any $r-1$ probabilities involved in terms of $p_{k, r}$. Leaving out the $r^{\text {th }}$ equation, we have the matrix representation of (7) as

$$
\boldsymbol{C} \boldsymbol{R}=-\left[(N-k) \rho_{r}+1\right] \mathbf{G} p_{k, r},
$$

where $\boldsymbol{C}$ is the $(r-1) \times(r-1)$ matrix

$$
\boldsymbol{C}=\left[c_{i j}\right]
$$

such that

$$
\begin{aligned}
c_{i j} & =\sigma_{i}\left[(N-k) \rho_{j}+1\right], i \neq \mathrm{j} \\
c_{i i} & =\left(\sigma_{i}-1\right)\left[(N-k) \rho_{i}+1\right],
\end{aligned}
$$

where

$$
\begin{aligned}
R^{T} & =\left[p_{k, 1}, p_{k, 2}, \ldots, p_{k, r-1}\right], \\
\mathbf{G}^{T} & =\left[\sigma_{1}, \sigma_{2}, \ldots, \sigma_{r-1}\right] .
\end{aligned}
$$

Now, $C^{-1}$ is given by

$$
C^{-1}=\left[c_{i j}^{*}\right]
$$

such that

$$
\begin{aligned}
c_{i j}^{*} & =\frac{-\sigma_{i}}{\sigma_{r}\left[(N-k) \rho_{i}+1\right]}, i \neq j, \\
c_{i i}^{*} & =\frac{-\left(\sigma_{i}+\sigma_{r}\right)}{\sigma_{r}\left[(N-k) \rho_{i}+1\right]},
\end{aligned}
$$

and

$$
p_{k, i}=\frac{\sigma_{i}\left[(N-k) \rho_{r}+1\right]}{\sigma_{r}\left[(N-k) \rho_{i}+1\right]} p_{k, r}, i=1,2, \ldots, r-1
$$

From (4) and (6) we obtain

$\left\{(N-n) \rho_{i}-\sigma_{i}+1\right\} p_{n, i}-\sigma_{i} \sum_{s \neq i}^{r} p_{n, s}=\mathrm{p}_{n+1, i}, n=2,3, \ldots k-1 ; i=1,2, \ldots, r$

which can be written in the matrix form as:

$$
A P=B
$$

where

$$
A=\left[a_{i j}\right]
$$

such that

$$
a_{i j}=-\sigma_{i}, i \neq j
$$




$$
\begin{aligned}
& a_{i i}=\left\{(N-n) \rho_{i}-\sigma_{i}+1\right\} \\
& P^{T}=\left[p_{n, 1}, p_{n, 2}, \ldots, p_{n, r}\right]
\end{aligned}
$$

and

$$
B^{T}=\left[p_{n+1,1}, p_{n+1,2}, \ldots, p_{n+1, r}\right],
$$

where $\mathrm{T}$ denotes the transpose of a matrix. Now, $A^{-1}$ is given by

$$
A^{-1}=\left[a_{i j}^{*}\right]
$$

where

$$
\begin{aligned}
& a_{i j}^{*}=\frac{\sigma_{i}}{D_{n}\left[(N-n) \rho_{i}+1\right]\left[(N-n) \rho_{j}+1\right]}, i \neq j, \\
& a_{i i}^{*}=\frac{1}{\left[(N-n) \rho_{i}+1\right]}+\frac{\sigma_{i}}{D_{n}\left[(N-n) \rho_{i}+1\right]^{2}},
\end{aligned}
$$

and

$$
D_{n}=1-\sum_{i=1}^{r} \frac{\sigma_{i}}{\left[(N-n) \rho_{i}+1\right]}, n=2,3, \ldots, k-1 .
$$

Using this value of $A^{-1}$ in (10), we get

$$
\begin{aligned}
p_{n, i}=\left[\frac{p_{n+1, i}}{\left[(N-n) \rho_{i}+1\right]}+\right. & \left.\frac{\sigma_{i}}{D_{n}\left[(N-n) \rho_{i}+1\right]} \sum_{s=1}^{r} \frac{p_{n+1, s}}{\left[(N-n) \rho_{s}+1\right]}\right], \\
& n=k-1, k-2, \ldots, 2 ; i=1,2, \ldots, r(11)
\end{aligned}
$$

From (1)-(3),we have

$$
\begin{array}{cc}
p_{1,0, i}=l_{i} \eta+g_{i} p_{1,1, i} ; & i=1(1) r \\
p_{0,1, i}=e_{i} \eta+f_{i} p_{1,1, i} ; & i=2(1) r \\
p_{0,0, i}=\frac{\mu_{1}}{N \lambda_{i}} p_{1,0, i}+\frac{\mu_{2}}{N \lambda_{i}} p_{0,1, i} ; & i=1(1) r
\end{array}
$$

where

$$
\begin{gathered}
g_{i}=\frac{\mu_{2}}{(\mathrm{~N}-1) \lambda_{i}+\mu_{1}}, \quad l_{i}=\frac{\sigma_{\mathrm{i}} \pi_{1}}{(\mathrm{~N}-1) \lambda_{i}+\mu_{1}}, \\
f_{i}=\frac{\mu_{1}}{(\mathrm{~N}-1) \lambda_{i}+\mu_{2}}, \quad e_{i}=\frac{\sigma_{\mathrm{i}} \pi_{2}}{(\mathrm{~N}-1) \lambda_{i}+\mu_{2}},
\end{gathered}
$$

and

$$
\eta=\frac{\sum_{s=1}^{r}\left(\mu_{1} g_{s}+\mu_{2} f_{s}\right) p_{2, s}}{1-\left\{\mu_{1} \sum_{s=1}^{r} l_{s}+\mu_{2} \sum_{s=1}^{r} e_{s}\right\}}
$$

Thus, we have expressed all probabilities for $n=0,1,2, \ldots, k ; i=1,2, \ldots, r$ in terms of one unknown probability, namely $p_{k, r}$. This unknown probability may now be computed by using the normalizing condition: $\sum_{n=0}^{k} \sum_{i=1}^{r} p_{n, i}=1$,

and hence all the probabilities are completely known in terms of the queue parameters. 
4-The main performance measures characteristic of machine interference problem are mentioned:

1-The expected number of down machines is

$$
L=\sum_{n=1}^{k} \sum_{s=1}^{r} n p_{n, s} .
$$

2-Machine efficiency ( the fraction of total production time on all machines) is

$$
U_{m}=1-\frac{L}{k}
$$

3-Average operator utilization

$$
U_{s}=\sum_{n=0}^{k} \sum_{s=1}^{r} \frac{n p_{n, s}}{c}+\sum_{n=c+1}^{k} \sum_{s=1}^{r} p_{n, s}
$$

or

$$
U_{s}=1-\sum_{n=0}^{k} \sum_{s=1}^{r}\left(1-\frac{n}{c}\right) p_{n, s}
$$

\section{5-Numerical Work}

The following example illustrates the method discussed above.

Example: In the system: $H_{r} / M / 2 / k / N$ letting $r=2, k=3$ and $N=4$, i.e., the queue : $\mathrm{H}_{2} / \mathrm{M} / 2 / 3 / 4$, the results are:

$$
\begin{aligned}
& p_{3,1}=a p_{3,2}, \quad p_{2,1}=b_{1} p_{3,2}, p_{2,2}=b_{2} p_{3,2}, \\
& p_{0,1,1}=e_{1} p_{3,2}, \quad p_{0,1,2}=e_{2} p_{3,2}, \\
& p_{1,0,1}=d_{1} p_{3,2}, \quad p_{1,0,2}=d_{2} p_{3,2} \\
& p_{0,0,1}=h_{1} p_{3,2}, \quad p_{0,0,2}=h_{2} p_{3,2}, \\
& \eta=\gamma p_{3,2}, \quad \gamma=\frac{\left(\mu_{1} g_{1}+\mu_{2} f_{1}\right) b_{1}+\left(\mu_{1} g_{2}+\mu_{2} f_{2}\right) b_{2}}{1-\left\{\mu_{1}\left(l_{1}+l_{2}\right)+\mu_{2}\left(l_{1}+l_{2}\right)\right\}},
\end{aligned}
$$

where

$$
\begin{gathered}
g_{i}=\frac{\mu_{2}}{3 \lambda_{i}+\mu_{1}}, \quad l_{i}=\frac{\sigma_{\mathrm{i}} \pi_{1}}{3 \lambda_{i}+\mu_{1}}, i=1,2 \\
f_{i}=\frac{\mu_{1}}{3 \lambda_{i}+\mu_{2}}, \quad e_{i}=\frac{\sigma_{\mathrm{i}} \pi_{2}}{3 \lambda_{i}+\mu_{2}} ; i=1,2, \\
a=\frac{\sigma_{1}\left(\rho_{2}+1\right)}{\sigma_{2}\left(\rho_{1}+1\right)}, \quad b_{1}=\frac{1}{2 \rho_{1}+1}\left[a+\frac{\sigma_{1}}{D_{2}}\left(\frac{a}{2 \rho_{1}+1}+\frac{1}{2 \rho_{2}+1}\right)\right], \\
b_{2}=\frac{1}{2 \rho_{2}+1}\left[a+\frac{\sigma_{2}}{D_{2}}\left(\frac{a}{2 \rho_{1}+1}+\frac{1}{2 \rho_{2}+1}\right)\right],
\end{gathered}
$$




$$
\begin{array}{ll}
d_{1}=\left(g_{1} b_{1}+l_{1} \gamma\right), & d_{2}=\left(g_{2} b_{2}+l_{2} \gamma\right), \\
c_{1}=\left(f_{1} b_{1}+e_{1} \gamma\right), & c_{2}=\left(f_{2} b_{2}+e_{2} \gamma\right), \\
h_{1}=\frac{\mu_{1} d_{1}+\mu_{2} e_{1}}{4 \lambda_{1}}, & h_{2}=\frac{\mu_{1} d_{2}+\mu_{2} c_{2}}{4 \lambda_{2}} .
\end{array}
$$

From the normalizing condition: $\sum_{n=0}^{3} \sum_{i=1}^{2} p_{n, i}=1$, we have

$$
p_{3,2}=\left[1+a+b_{1}+b_{2}+d_{1}+d_{2}+c_{1}+c_{2}+h_{1}+h_{2}\right]^{-1} .
$$

The expected number of machines in the system is

$$
\begin{gathered}
L=\sum_{n=1}^{3} \sum_{s=1}^{2} n p_{n, s} \\
=\left\{d_{1}+d_{2}+c_{1}+c_{2}+2\left(b_{1}+b_{2}\right)+3(a+1)\right\} p_{3,2},
\end{gathered}
$$

Machine efficiency ( the fraction of total production time on all machines) is

$$
U_{m}=1-\frac{L}{3}
$$

Average operator utilization

$$
\begin{aligned}
& U_{s}=\sum_{n=0}^{2} \sum_{s=1}^{2} \frac{n p_{n, s}}{2}+\sum_{s=1}^{2} p_{3, s} \\
& U_{s}=1-\left\{\left(h_{1}+h_{1}\right)+\frac{1}{2}\left(d_{1}+d_{2}+c_{1}+c_{2}\right)\right\} p_{3,2} .
\end{aligned}
$$

\section{6- special Cases}

Some queuing systems can be obtained as special cases of this model:

(i) If $r=1$ we get the model : M/M/2/ $k / \mathrm{N}$, which was studied by Shawky [10] at $\alpha=0$ and $\beta=1$

(ii) If . $k=N \rightarrow \infty, r=1, \pi_{1}=1$, and $\pi_{2}=0$ we obtain the queue $\mathrm{M} / \mathrm{M} / 2$ which was studied by Singh[11].

(iii) If $k=N=m, \mu_{1}=\mu_{2}, r=1$ and $\pi_{1}=\pi_{2}=1 / 2$,we get the homogeneous repairmen model : M/M/2/ $m$ / $m$ which was discussed by White et al. [13], Gross and Harris [7] and Bunday [2].

\section{7-Conclusion}

In this paper, the machine interference model: $H_{r} / M / 2 / k / N$ is studied with two heterogeneous repairmen. The recurrence relations that give all the probabilities in terms of $\mathrm{P}_{k, r}$ are derived. We illustrated the method by a numerical example and deduced the expected number of units in the system and machine efficiency, also the average operator utilization are derived. Some special cases are obtained. 


\section{References}

[1] Abou-Al-ATa,(1992). An interval Hyper exponential Machine interference with Balking and Reneging. Microelectronic Relab .vol.32,no. 9,pp. 1251-1256.

[2] B. D.Bunday, (1996). An introduction to queueing theory. Jphn Wiley, New York.

[3] P. C. George and A. G. Simon (1980). Approximations in the Multi-Server Queue with Hyper- Exponential Inter-arrival Times and Exponential Service Times . J. Ops. Res. Soc.vol. 31,pp.57-62.

[4] J. K. Goyal (1967) . Queues with Hyper-Poisson Arrivals and Bulk Exponential Service. Metrika 11, pp. 157-167.

[5] S. K. Gupta,(1967). Queues with Hyper-Poisson Input and Exponential Service Time Distribution with State Dependent Arrival and Service Rate. Ops. Res., 15, pp. 847-856.

[6] H., Gumble, (1960). Waiting Lines with Heterogeneous Servers. Ops. Res., pp. 504-511.

[ 7] D. Gross and C. M. Harris,(1974). Fundamentals of queueing theory. John Wiley, New York.

[8] B. Krishnamoorthi, (1963), On Poisson Queue with Two Heterogeneous Serves. Ops. Res., 11, PP.321-330,

[9] A.I Shawky (2001). An Inter-Arrival Hyper- Exponential Machine Interference Model :

$H_{r} / M / c / k / N$ with Balking and Reneging. Comm. Korean Math. Soc. 16, no.4, pp. 656-666.

[10] A. I. Shawky (2004). On Poisson Machine Interference Model: $M / M / 2 / k / N$ with Balking, Reneging and Heterogeneous Repairmen. common., Fas. Sei., Univ. Ank. , Series AL: Mathematics and statistics, vol. 53,no. 2,pp. 7-12.

[11] V. P. Singh,(1970). Two-Server Markovian Queues with Balking: Heterogeneous Vs, homogeneous Servers. Ops. Res.,no.18, pp. 145-159

[12] T. L., Saaty, (1960). Time Dependent Solution of the Many -Server Poisson Queue. Ops. Res. ,Vol. 8, pp. 768-771.

[13] J. A. White, J. W. Schmidt and G. K. Benntt, (1975). Analysis of queueing system. Academic press, New York. 